\section{Evaluation of SRAP markers for mapping of Pisum sativum L.}

\author{
María Fernanda Guindon ${ }^{1}$, Eugenia Martin ${ }^{1 *}$, Aldana Zayas ${ }^{1}$, \\ Enrique Cointry ${ }^{1}$ and Vanina Cravero ${ }^{1}$
}

\begin{abstract}
Linkage maps have become important tools for genetic studies. With the aim of evaluating the SRAP (sequence-related amplified polymorphism) technique for linkage mapping in Pisum sativum L., a $F_{2}$ mapping population derived from an initial cross between cvs. DDR11 and Zav25 was generated. A total of 25 SRAP primer combinations were evaluated in $45 F_{2}$ plants and both parental lines, generating 208 polymorphic bands/markers. The markers were analyzed by the chi-square goodness-of-fit test to check the expected Mendelian segregation ratio. The resulting linkage map consists of 112 genetic markers distributed in 7 linkage groups (LGs), covering a total of $528.8 \mathrm{cM}$. The length of the LGs ranged from 47.6 to $144.3 \mathrm{cM}$ (mean $75.54 \mathrm{cM}$ ), with 9 to 34 markers. The linkage map developed in this study indicates that the SRAP marker system could be applied to mapping studies of pea.
\end{abstract}

Key words: Pea, plant breeding, linkage map, $F_{2}$ population, molecular markers.

\section{INTRODUCTION}

Pea (Pisum sativum L.) is an autogamous, annual cool-season legume originated from areas in the Middle East, in the East of the Caucasus, Iran and Afghanistan, and West of the Mediterranean basin (Smýkal et al. 2011). Its genome is organized in seven chromosome pairs $(2 n=2 x=14)$, and the haploid size estimated at $4.45 \mathrm{~Gb}$ (Smýkal et al. 2012). Peas were an important source of animal and human food for many centuries. The species is rich in protein, slowly digestible starch, soluble sugars, fiber, minerals, and vitamins (Dahl et al. 2012). The global dry pea production averages 10 million tonnes a year. Argentina is one of the top exporting countries, eighth in the global ranking between 2008 and 2011 (FAO 2012). The rising world population will require increased crop production. Moreover, some researchers suggest that the current rate of increase in crop yields will not be enough to meet this demand (Tester and Langridge 2010). Therefore, plant breeding programs are needed to further raise crop yields. In this context, linkage mapping will be useful to maximize the success probability. Genetic linkage maps are powerful tools for genetic research and breeding of plants. The linkage maps are the first step in: 1) the analysis of qualitative and quantitative traits; 2 ) the introgression of desirable genes and quantitative trait loci (QTLs); 3) positional or map-based cloning of genes responsible for economically important traits (Semagn et al. 2006). Different kinds of markers, such as simple sequence repeats (SSR; Loridon et al. 2005), single nucleotide polymorphisms (SNP; Deulvot et al. 2010), inter simple sequence repeats (ISSR; Mishra et al. 2009), and sequence tagged sites (STS; Barilli et al.
Crop Breeding and Applied Biotechnology 16: 182-188, 2016 Brazilian Society of Plant Breeding. Printed in Brazil http://dx.doi.org/10.1590/1984$70332016 v 16 n 3 a 28$

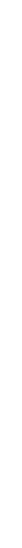

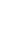

.


2010) have been used to develop moderate density linkage maps in pea. Several markers were common in different maps corresponding to different crosses; this allowed an integration of these maps (Loridon et al. 2005, Aubert et al. 2006), as well as the development of consensus linkage maps for the species (Weeden et al. 1998, Bordat et al. 2011).

In this study, we proposed the use of the sequence-related amplified polymorphism (SRAP) technique (Li and Quiros 2001) to generate a number of markers distributed across all pea chromosomes. Since its development, SRAP has been employed in a wide range of plant species for genetic diversity estimation (Cravero et al. 2007, Espósito et al. 2007, Aneja et al. 2012), gene tagging (Martin et al. 2008, Zhang et al. 2010), and map construction (Lin et al. 2005, Sun et al. 2007, Wang et al. 2008, Martin et al. 2013). The aim of the current study was to evaluate the usefulness of SRAP markers in the development of a genetic linkage map of Pisum sativum L.

\section{MATERIAL AND METHODS}

\section{Plant material}

The $F_{2}$ mapping population was derived from an initial cross between the cvs. DDR11 and Zav25. The latter is an experimental line obtained from the IICAR-CONICET breeding program (Espósito et al. 2007). For most yield-related traits, such as number of pods and seeds per plot, the values of 'DDR11' are lower than those of 'Zav25'.

Both parents and 45 plants of the $F_{2}$ population were sown in an experimental field of Universidad Nacional de Rosario (lat $33^{\circ} 1^{\prime} \mathrm{S}$, long $60^{\circ} 53^{\prime} \mathrm{W}$ ) in the winter of 2012, in a completely randomized design (inter-row spacing 70 $\mathrm{cm}$, plant spacing $10 \mathrm{~cm}$ ).

\section{DNA extraction}

The genomic DNA of each $F_{2}$ plant and both parents was isolated from fresh leaves by the CTAB method described by Doyle and Doyle (1990), with the following modifications: after DNA precipitation, the samples were stored at $-20^{\circ} \mathrm{C}$ for $30 \mathrm{~min}$, the two washes of the final step were performed with ethanol $70 \%$, and the resulting pellet was resuspended in distilled water.

After RNAase-treatment, each DNA sample was quantified using agarose gel electrophoresis $(1 \% \mathrm{w} / \mathrm{v})$ and comparison of band intensity with the standard $\lambda$ DNA $\left(76 \mathrm{ng} \mathrm{L}^{-1}\right)$. The hybrid origin of each plant was checked using two microsatellite markers SSR: PSMPSAA135 and PSMPSAA205 (Tar'an et al. 2005), which were contrasting in both parental lines.

\section{SRAP genotypic analysis}

The $F_{2}$ population was genotyped using 25 SRAP primer combinations generated from five forward and five reverse primers developed by Li and Quiros (2001) (Table 1). The primers are 17-18 bases long and have a core sequence, which includes 10-11 non-specific bases at the $5^{\prime}$ end and sequence CCGG in the forward and AATT in the reverse primer. The core sequence is followed by three selective nucleotides at the $3^{\prime}$ end of each primer. The primers were selected based on the results of Espósito et al. (2007) in a characterization of pea accessions with this type of molecular markers.

Polymerase chain reactions were carried out in a final volume of $20 \mu \mathrm{L}$ containing $15 \mathrm{ng}$ genomic DNA, $0.2 \mathrm{mM}$ dNTPs, $1.5 \mathrm{mM} \mathrm{MgCl}_{2}, 0.5 \mu \mathrm{M}$ of each primer, $1 \mathrm{X}$ Taq buffer (Invitrogen, California, USA), and $1 \mathrm{U}$ of Taq recombinant polymerase (Invitrogen). Samples were subjected to the following thermal profile: 5 min denaturing at $94^{\circ} \mathrm{C}$ and five cycles of three steps: 1 min denaturing at $94^{\circ} \mathrm{C}, 1 \mathrm{~min}$ annealing at $35^{\circ} \mathrm{C}$, and 1 min elongation at $72{ }^{\circ} \mathrm{C}$; for the following 35 cycles, annealing temperature was elevated to $50^{\circ} \mathrm{C}$ with a final elongation step of $10 \mathrm{~min}$ at $72{ }^{\circ} \mathrm{C}$.

Table 1. Primer sequences used for SRAP (Sequence-related amplified polymorphism) analysis

\begin{tabular}{ll}
\hline Forward & \multicolumn{1}{c}{ Reverse } \\
\hline me1 5'-TGAGTCCAAACCGGATA-3' & em1 5'-GACTGCGTACGAATTAAT-3' \\
me2 5'-TGAGTCCAAACCGGAGC-3' & em2 5'-GACTGCGTACGAATTTGC-3' \\
me3 5'-TGAGTCCAAACCGGAAT-3' & em3 5'-GACTGCGTACGAATTGAC-3' \\
me4 5'-TGAGTCCAAACCGGACC-3' & em4 5'-GACTGCGTACGAATTTGA-3' \\
me5 5'-TGAGTCCAAACCGGAAG-3' & em5 5'-GACTGCGTACGAATTAAC-3' \\
\hline
\end{tabular}


The resulting amplicons were separated on $6 \%(\mathrm{w} / \mathrm{v})$ denaturing polyacrylamide gels and then visualized by silver staining (Bassam et al. 1991). The SRAP fragments were treated as dominant markers. Each marker was labeled according to the primer combination used for its generation plus the estimated amplicon size.

\section{Linkage analysis and linkage map construction}

Linkage analyses were performed using JoinMap v4 (van Ooijen 2006). Each segregating marker was tested for deviations from the expected 3:1 segregation ratio using Chi-square tests. Markers with Mendelian segregation $\left(x^{2} \leq x_{\alpha=0.1}^{2}\right)$ or with minor distortion $\left(x_{\alpha=0.1}^{2}<x^{2} \leq x_{\alpha=0.01}^{2}\right)$ were used for the construction of the linkage map. Markers with highly distorted segregation $\left(x^{2}>x_{\alpha=0.01}^{2}\right)$ were included in a second step of mapping only when their presence did not affect the local marker order. Linkage groups (LGs) were established at a minimum LOD (logarithm of odds) value of 3.0. The marker order for each $L G$ was determined at $L O D=1.0, R E C=0.40$ and Jump $=5$. Recombination values were converted to genetic distances using the Kosambi (1994) mapping function. Linkage groups were numbered sequentially according to their length in cM. Linkage maps were drawn using MapChart 2.2 software (Voorrips 2002).

\section{RESULTS AND DISCUSSION}

Since the development of molecular marker techniques, the number of marker loci identified on genetic maps is increasing at a high rate. The SRAP marker system designed by Li and Quiros (2001) is a simple and efficient technique. It has several advantages over other molecular markers, namely its simplicity and reasonable throughput rate. It also allows easy isolation of bands for sequencing and, most importantly, it targets ORFs (open reading frames). Elsewhere, SRAPs were established as a powerful tool for construction of genetic linkage maps, e.g., of Brassica (Li and Quiros 2001, Sun et al. 2007), Gossypium (Lin et al. 2005, Yu et al. 2007), Cucumis melo L. (Wang et al. 2008), and more recently of Cynara cardunculus (Martin et al. 2013). Our study presents the first application of SRAP markers for the construction of a linkage map of $P$. sativum $\mathrm{L}$.

The parental lines to develop the mapping population ('DDR11' and 'Zav25') were selected based on observations of Espósito et al. (2007), who reported that these lines are divergent at the morphological (Euclidean distance $=0.47$ ) and molecular levels (Dice distance $=0.66$ ) and that they were grouped separately by hierarchical cluster analysis.

A total of 25 SRAP primer combinations were used of which 23 pairs were amplified. Most combinations produced clear bands without overlapping, but in some cases the scoring of the markers was somewhat cumbersome, because of the high number of bands and their different intensities, and the presence of minor bands in some plants. The number of fragments amplified by each primer combination ranged from 3 (Me1-Em1; Me1-Em4) to 24 (Me3-Em2), with an average of 9.96. A total of 208 polymorphic bands (PB) were generated. The most polymorphic primer combination was Me2-Em3, with 18 PB (Table 2).

The markers proved efficient for genetic studies in pea, producing an average of $8.32 \mathrm{~PB} /$ primer combination. This value is similar to those of the other species, where 3 to 14 bands per primer combination were reported ( $\mathrm{Li}$ and Quiros 2001, Lin et al. 2003, Sun et al. 2007, Yu et al. 2007, Wang et al. 2008, Martin et al. 2013). Since one primer combination may detect a high number of polymorphic loci,

Table 2. Number of bands and polymorphic bands generated by SRAP primer combinations

\begin{tabular}{|c|c|c|}
\hline Combination & Bands & Polymorphic bands \\
\hline Me1-Em1 & 3 & 3 \\
\hline Me1-Em2 & 9 & 9 \\
\hline Me1-Em3 & 5 & 5 \\
\hline Me1-Em4 & 3 & 2 \\
\hline Me1-Em5 & 15 & 14 \\
\hline Me2-Em1 & 8 & 8 \\
\hline Me2-Em2 & 9 & 9 \\
\hline Me2-Em3 & 18 & 18 \\
\hline Me2-Em4 & 16 & 14 \\
\hline Me2-Em5 & 13 & 9 \\
\hline Me3-Em1 & 4 & 1 \\
\hline Me3-Em2 & 24 & 16 \\
\hline Me3-Em3 & 8 & 8 \\
\hline Me3-Em4 & 10 & 8 \\
\hline Me3-Em5 & 8 & 7 \\
\hline Me4-Em1 & 0 & 0 \\
\hline Me4-Em2 & 10 & 7 \\
\hline Me4-Em3 & 6 & 6 \\
\hline Me4-Em4 & 17 & 12 \\
\hline Me4-Em5 & 5 & 5 \\
\hline Me5-Em1 & 0 & 0 \\
\hline Me5-Em2 & 6 & 3 \\
\hline Me5-Em3 & 16 & 16 \\
\hline Me5-Em4 & 18 & 14 \\
\hline Me5-Em5 & 18 & 14 \\
\hline Total & 249 & 208 \\
\hline
\end{tabular}


Evaluation of SRAP markers for mapping of Pisum sativum $\mathrm{L}$.

Table 3. Characteristics of the linkage groups (LG) generated by SRAP markers

\begin{tabular}{|c|c|c|c|c|c|c|}
\hline LG & Number of markers & $\begin{array}{l}\text { Number of highly } \\
\text { distorted markers }\end{array}$ & Size & $\begin{array}{l}\text { Smallest distance } \\
\text { between markers }\end{array}$ & $\begin{array}{l}\text { Highest distance } \\
\text { between markers }\end{array}$ & $\begin{array}{l}\text { Average distance } \\
\text { between markers }\end{array}$ \\
\hline 1 & 34 & 6 & 144.309 & 0.031 & 25.392 & 4.244 \\
\hline 3 & 18 & 1 & 79.119 & 0.204 & 16.06 & 4.395 \\
\hline 4 & 10 & 1 & 61.401 & 1.608 & 17.801 & 6.140 \\
\hline 7 & 9 & 2 & 47.601 & 2.207 & 15.1 & 5.289 \\
\hline
\end{tabular}

this technique can be used to construct ultra-dense genetic maps. Furthermore, SRAP markers can be combined with next-generation techniques to enhance their capacity and effectiveness. Li et al. (2011) combined SRAP with Illumina/ Solexa sequencing to directly integrate genetic loci in the B. rapa genetic map based on paired-end Solexa sequencing. Results of the SRAP technique obtained in this way may prove invaluable for QTL analysis and map-based cloning.

Chi-square analysis of the 208 loci revealed that 116 loci ( 55.8\%) were consistent with the expected Mendelian 3:1 segregation ratio $\left(x^{2} \leq x_{\alpha=0.1}^{2}\right)$, the distortion of 37 loci ( 17.8\%) was minor $\left(x_{\alpha=0.1}^{2}<x^{2} \leq x_{\alpha=0.01}^{2}\right)$ and 55 loci $(\sim 26.4 \%)$ were highly distorted $\left(x^{2}>x_{\alpha=0.01}^{2}\right)$.

Initially, the 153 markers with Mendelian or slightly distorted segregation were used for the construction of linkage groups (LG), using a minimum LOD value of 3.0 and the mapping parameters $\operatorname{Rec}=0.40, L O D=1.0$, and Jump $=5$. Under

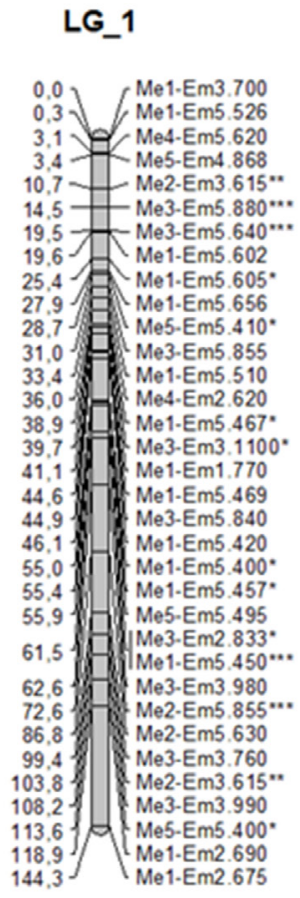

$$
\text { LG_2 }
$$

LG_3

LG_4

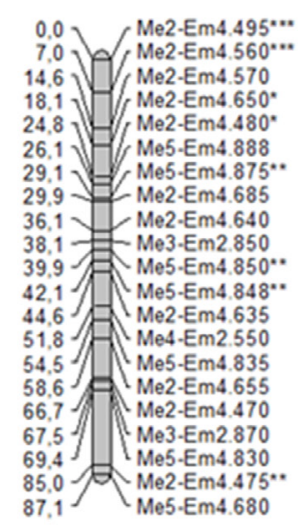

$$
\text { LG_5 }
$$

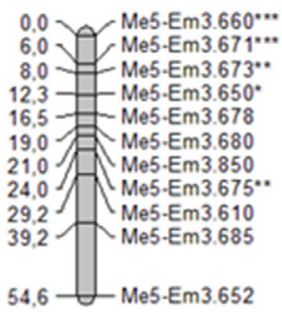

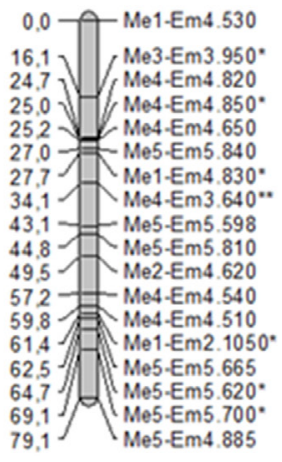

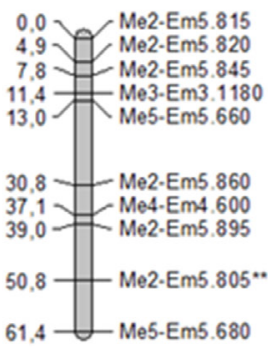

LG_6

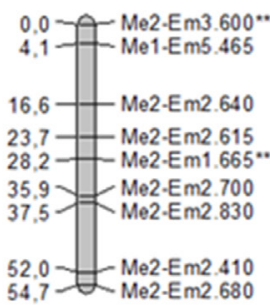

\section{LG_7}

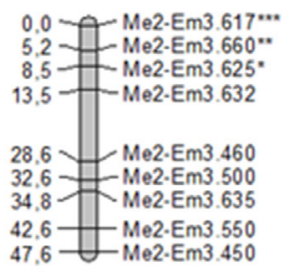

Figure 1. Genetic linkage map of pea. Marker names are shown on the right of each LG (linkage group) and map distances (in cM) on the left. Markers with significant levels of segregation distortion are indicated by asterisks (minor distortion * $0.05 \geq P>0.01$, highly distorted segregation: $* * 0.01 \geq \mathrm{P}>0.001, * * * \mathrm{P} \leq 0.001)$. 
these conditions, an initial framework map with 62 loci was constructed. Then the framework order was fixed and a second round of mapping performed, including markers with distorted segregation. These markers were only included in the final map if their presence did not alter the surrounding marker order in a given linkage group. By this step, we incorporated 29 Mendelian markers and 22 distorted markers in the previously established linkage groups. This strategy ensures the accuracy and a high coverage of the final map. Similar strategies were successfully used for linkage mapping in different species, e g., of olive (Khadari et al. 2010), globe artichoke and cardoon (Martin et al. 2013), lentil (Verma et al. 2015), poplar (Zhou et al. 2015), and wheat (Li et al. 2015).

The resulting map comprises 112 loci distributed over seven linkage groups (LGs), which is equal to the haploid number of chromosomes in the pea genome (Figure 1). The overall map length was $528.8 \mathrm{cM}$ and the mean inter-marker distance $4.72 \mathrm{cM}$. The LG length varied from $47.6 \mathrm{cM}$ to $144.3 \mathrm{cM}$. The number of markers included in each $\mathrm{LG}$ ranged from 9 to 34 (Table 3). A total of 22 distorted markers (19\%) were included in the genetic map.

Although several linkage maps for pea have been developed using different kinds of markers, this is the first linkage map of this species constructed with SRAP markers. The length of our map ( $528.8 \mathrm{cM}$ ) is shorter than that of previous ones generated with other molecular markers, which covered 1430, 1458, and $1283 \mathrm{cM}$, respectively (Loridon et al. 2005, Aubert et al. 2006, Barilli et al. 2010). All these genetic maps were constructed using mapping populations with different sizes, derived from different crosses. Both, the size of the mapping population and their origin affect the marker coverage and map length because an increasing divergence between parents generates a greater number of possible recombinations and the possibility of finding recombinant plants is higher when populations are large. Then, the small size of our $\mathrm{F}_{2}$ population ( 45 plants) could be the cause of the smaller size of the map obtained in this study (Ferreira et al. 2006). To enhance accuracy and reduce the statistical error, a great number of plants should be evaluated. On the other hand, the large number of unlinked markers with Mendelian or slightly distorted segregation (62) reflects the need to enrich this map with additional markers to cover the entire genome.

\section{CONCLUSIONS}

The linkage map generated in this study provided basic information for assistance of future molecular marker application in the local breeding program of Pisum sativum L. Since pea has no reference genome, molecular markers that do not require sequence information must be evaluated. In this context, sequence-related amplified polymorphism (SRAP) represents an efficient tool for genetic analysis of pea even though the proposed linkage map was only partly saturated. For the first time, SRAP markers were applied in this study to develop a linkage map of pea. Moreover, since these markers target coding regions of the genome, they can potentially identify markers with inherent biological significance. Additional markers are required to expand the coverage of this map for QTL analysis. The segregating population used to develop this linkage map is currently being phenotyped for yield-related traits to detect QTLs associated to this character.

\section{ACKNOWLEDGEMENTS}

This research was supported by Consejo Nacional de Investigaciones Científicas y Técnicas (CONICET, Argentina) and Fondo para la Investigación Científica y Tecnológica (FONCyT, Argentina).

\section{REFERENCES}

Aneja B, Yadav NR, Chawla V and Yadav RC (2012) Sequence-related amplified polymorphism (SRAP) molecular marker system and its applications in crop improvement. Molecular Breeding 30: 16351648.

Aubert G, Morin J, Jacquin F, Loridon K, Quillet MC, Petit A, Rameau C, Lejeune-Hénaut I, Huguet T and Burstin J (2006) Functional mapping in pea, as an aid to the candidate gene selection and for investigating synteny with the model legume Medicago truncatula. Theoretical and Applied Genetics 112: 1024-1041.
Barilli E, Satovic Z, Rubiales D and Torres AM (2010) Mapping of quantitative trait loci controlling partial resistance against rust incited by Uromyces pisi (Pers.) Wint. in a Pisum fulvum L. intraspecific cross. Euphytica 175: 151-159.

Bassam BJ, Caetanoanolles G and Gresshoff PM (1991) Fast and sensitive silver staining of DNA in polyacrylamide gels. Analytical Biochemistry 196: 80-83.

Bordat A, Savois V, Nicolas M, Salse J, Chauveau A, Burgeois M, Potier J, Houtin H, Rond C, Murat F, Marget P, Aubert G and Burstin J (2011) Translational genomics in legumes allowed Placing in silico 5460 unigenes on the pea functional map and identified candidate genes 
in Pisum sativum L. G3: Genes, Genomes, Genetics 1: 93-103.

Cravero VP, Martin EA and Cointry EL (2007) Genetic diversity in Cynara Cardunculus determined by sequence-related amplified polymorphism markers. Journal of the American Society for Horticultural Science 132: 208-212.

Dahl W, Foster L and Tyler R (2012) Review of the health benefits of peas (Pisum sativum L.) British Journal of Nutrition 108: 3-10.

Deulvot C, Charrel H, Marty A, Jacquin F, Donnadieu C, Lejeune-Hénaut I, Burstin J and Aubert G (2010) Highly-multiplexed SNP genotyping for genetic mapping and germplasm diversity studies in pea. BMC Genomics 11: 468-478.

Doyle JJ and Doyle JL (1990) Isolation of plant DNA from fresh tissue. Focus 12: 149-151.

Espósito MA, Martin EA, Cravero VP and Cointry EL (2007) Characterization of pea accessions by SRAP's markers. Scientia Horticulturae 113: 329-335.

FAO (2012) The Statistics Division of the FAO. Food and Agriculture Organization of the United Nations. Available at <http://faostat. fao.org/site/567/default.aspx\#ancor.> Accessed on June 6, 2014.

Ferreira A, Flores da Silva M, Silva LC and Cruz CD (2006) Estimating the effects of population size and type on the accuracy of genetic maps. Genetics and Molecular Biology 29: 187-192.

Khadari B, El Aabidine AZ, Grout C, Sadok IB, Doligez A, Moutier N, Santoni $S$ and Costes E (2010) A genetic linkage map of olive based on amplified fragment length polymorphism, intersimple sequence repeat and simple sequence repeat markers. Journal of the American Society for Horticultural Science 135: 548-555.

Li G and Quiros CF (2001) Sequence-related amplified polymorphism (SRAP), a new marker system based on a simple PCR reaction: its application to mapping and gene tagging in Brassica. Theoretical and Applied Genetics 103: 455-461.

Li W, Zhang, J, Mou Y, Geng J, McVetty P, Hu S and Li G (2011) Integration of Solexa sequences on an ultradense genetic map in Brassica rapa L. BMC Genomics 12: 249-263

Li C, Bai G, Chao S and Wang Z (2015) A high-density SNP and SSR consensus map reveals segregation distortion regions in wheat. . BioMed Research International 2015: Article ID 830618, 10 pages.

Lin Z, Zhang X, Nie Y, He D and Wu M (2003) Construction of a genetic linkage map for cotton based on SRAP. Chinese Science Bulletin 48: 2064-2068.

Lin Z, He D, Zhang X, Nie Y, Guo X and Feng C (2005) Linkage map construction and mapping QTL for cotton fibre quality using SRAP, SSR and RAPD. Plant Breeding 124: 180-187.

Loridon K, McPhee K, Morin J, Dubreuil P, Pilet-Nayel ML, Aubert G, Rameau C, Baranger A, Coyne C, Lejeune-He naut I and Burstin J (2005) Microsatellite marker polymorphism and mapping in pea (Pisum sativum L.). Theoretical and Applied Genetics 111: 10221031.
Martin E, Cravero V, Espósito M, López Anido F, Milanesi L and Cointry E (2008) Identification of markers linked to agronomic traits in globe artichoke. Australian Journal of Crop Science 1: 43-46.

Martin E, Cravero V, Portis E, Scaglione D, Acquaviva E and Cointry E (2013) New genetic maps for globe artichoke and wild cardoon and their alignment with an SSR-based consensus map. Molecular Breeding 32: 177-187

Mishra RK, Kumar A, Chaudhary S and Kumar S (2009) Mapping of the multifoliate pinna ( $\mathrm{mfp}$ ) leaf-blade morphology mutation in grain pea Pisum sativum. Journal of Genetics 88: 227-232.

Semagn K, Bjornstad A and Ndjiondjop MN (2006) Principles, requirements and prospects of genetic mapping in plants. Africal Journal of Biotechnology 5: 2569-2587.

Smýkal P, Kenicer G, Flavell AJ, Corander J, Kosterin O, Redden RJ, Ford R, Coyne CJ, Maxted N, Ambrose MJ and Ellis NTH (2011) Phylogeny, phylogeography and genetic diversity of the Pisum genus. Plant Genetic Resources: Characterization and Utilization 9: 4-18.

Smýkal P, Aubert G, Burstin J, Coyne CJ, Ellis NTH, Flavell AJ, Ford R, Hýbl M, Macas J, Neumann P, McPhee KE, Redden RJ, Rubiales D, Weller $\mathrm{JL}$ and Warkentin TD (2012) Pea (Pisum sativum L.) in the genomic era. Agronomy 2: 74-115.

Sun Z, Wang Z, Tu J, Zhang J, Yu F, McVetty P and Li G (2007) An ultradense genetic recombination map for Brassica napus, consisting of 13551 SRAP markers. Theoretical and Applied Genetics 114: 1305-1317.

Tar'an B, Zhang C, Warkentin T, Tullu A and Vandenberg A (2005) Genetic diversity among varieties and wild species accessions of pea (Pisum sativum L.) based on molecular markers, and morphological and physiological characters. Genome 48: 257-272.

Tester M and Langridge P (2010) Breeding technologies to increase crop production in a changing world. Science 327: 818-822.

van Ooijen JW (2006) Software for the calculation of genetic linkage maps in experimental populations. Demo Version. Kyazma B.V., Wageningen. Available at <https://www.kyazma.nl/index.php/ JoinMap/Evaluate/> Accessed in June 2015.

Verma P, Goyal R, Chahota RK, Sharma TR, Abdin MZ and Bhatia S (2015) Construction of a genetic linkage map and identification of QTLs for seed weight and seed size traits in lentil (Lens culinaris Medik.). PLoS ONE 10. doi:10.1371/journal.pone.0139666.

Voorrips RE (2002) MapChart: Software for the graphical presentation of linkage maps and QTLs. The Journal of Heredity 93: 77-78.

Wang J, Yao J and Li W (2008) Construction of a molecular map for melon (Cucumis melo L.) based on SRAP. Frontiers of Agriculture in China 2: 451-455.

Weeden NF, Ellis THN, Timmerman-Vaughan GM, Swiecicki WK, Rozov SM and Berdnikov VA (1998) A consensus linkage map for Pisum sativum. Journal of Heredity 83: 123-129.

Yu J, Yu S, Lu C, Wang W, Fan S, Song M, Lin Z, Zhang J and Zhang X (2007) High-density linkage map of cultivated allotetraploid cotton based 
on SSR, TRAP, SRAP and AFLP markers. Journal of Integrative Plant Biology 49: 716-724.

Zhang W, He H, Guan Y, Du H, Yuan L, Li Z, Yao D, Pan J and Cai R (2010) Identification and mapping of molecular markers linked to the tuberculate fruit gene in the cucumber (Cucumis sativus L.)
Theoretical and Applied Genetics 120: 645-654.

Zhou W, Tang Z, Hou J, Hu N and Yin T (2015) Genetic map construction and detection of genetic loci underlying segregation distortion in an intraspecific cross of Populus deltoides. PLoS ONE 10. doi:10.1371/ journal.pone.0126077. 